\title{
Updates in pharmacotherapy of heart failure with reduced ejection fraction
}

\author{
Clifton Espinoza, Haider Alkhateeb, Tariq Siddiqui
}

Division of Cardiovascular Diseases, Department of Internal Medicine, Texas Tech University Health Science Center El Paso, El Paso, TX, USA Contributions: (I) Conception and design: All authors; (II) Administrative support: All authors (III) Provision of study materials or patients: C Espinoza; (IV) Collection and assembly of data: All authors; (V) Data analysis and interpretation: All authors; (VI) Manuscript writing: All authors; (VII) Final approval of manuscript: All authors.

Correspondence to: Clifton Espinoza, MD. Division of Cardiovascular Diseases, Department of Internal Medicine, Texas Tech University Health Science Center El Paso, 4800 Alberta Avenue, El Paso, Texas 79905, USA. Email: Clifton.Espinoza@ttuhsc.edu.

\begin{abstract}
Heart failure is a common entity encountered in healthcare with a vast socioeconomic impact. Recent advances in pharmacotherapy have led to the development of novel therapies with mortality benefits, improvement in heart failure symptoms and hospitalizations. This article is intended to explore those newer pharmacotherapies and summarize the evidence behind guideline directed medical therapy (GDMT) for heart failure with reduced ejection fraction (HFrEF). It has been several years since any significant advances in pharmacotherapy of heart failure have resulted in survival benefit. Angiotensin-neprilysin inhibitors through the PARADIGM-HF and PIONEER-HF trials have shown mortality benefits and a reduction in heart failure hospitalizations and are considered landmark trials in heart failure. Vericiguat is an oral guanylate cyclase stimulator that through the recent VICTORIA trial showed a $10 \%$ relative difference in death from cardiovascular cause or hospitalization for heart failure. The sodium-glucose transport protein 2 (SGLT2) inhibitors are another class of medications that have shown promise in the treatment of patients with HFrEF and diabetes mellitus. The CANVAS and EMPA-REG OUTCOME trials showed the potential benefit of SGLT2 inhibitors on cardiovascular mortality, DECLARE-TIMI 58 trial showed that treatment with dapagliflozin reduced the risk of cardiovascular death or hospitalization for heart failure to a greater extent in patients with reduced ejection fraction (EF). Although novel pharmacotherapy is the current focus of intense research, there have been numerous studies on potential benefit of iron supplementation in ferropenic patients with heart failure. Another rapidly expanding area of research in the realm of heart failure is precision medicine and its impact on the development, progression, and treatment of heart failure. The field of heart failure is dynamic and with the influx of data from recent and ongoing trials, newer therapies with morbidity and mortality benefits in HFrEF are now available, nonetheless, much work is still needed.
\end{abstract}

Keywords: Heart failure with reduced ejection fraction (HFrEF); systolic heart failure; ivabradine; angiotensin receptor neprilysin inhibitor (ARNI); vericiguat; SGLT2 inhibitors; iron replacement

Submitted Jun 11, 2020. Accepted for publication Aug 28, 2020.

doi: 10.21037/atm-20-4640

View this article at: http://dx.doi.org/10.21037/atm-20-4640

Congestive heart failure (HF) is a clinical syndrome whereby a heart with systolic, diastolic, or combined dysfunction is no longer able to compensate resulting in a state of volume overload (1). It affects an estimated 6.2 million American $\geq 20$ years of age and $1-2 \%$ of the adult population in developed countries with an estimated
46\% increase in prevalence between 2012 and $2030(1,2)$.

This volume overload in of itself causes increased intracardiac filling pressures and congestion of blood in structures upstream to the heart such as the lungs resulting in pulmonary edema, inferior vena cava (IVC) and superior vena cava (SVC) resulting in jugular venous distention (JVD) 
and hepatic tenderness respectively. Elevated left ventricular (LV) filling pressure further decreases cardiac output resulting in decreased perfusion to vital organs such as the kidneys resulting in cardiorenal syndrome, this further lead to retention of salt within the kidneys and subsequently exacerbates an already congested vasculature (3). It is these physiologic feedback loops designed to maintain intravascular volume and organ perfusion that result in an endless loop that eventually leads to a decompensated state. Guideline directed medical therapy function in one or several of these feedback loops to break the cycle of decompensation.

The goal of this review article is to briefly review the present guideline-directed medical management (GDMT) for systolic HF which have shown to improve LV function and decrease mortality with emphasis on some of the newest available therapies.

\section{Hydralazine/isosorbide dinitrate combination}

One of the earliest trials to evaluate pharmacotherapy in HF patients was the V-HEFT trial published in 1986. V-HEFT randomized 642 patients with symptomatic chronic compensated systolic HF with left ventricular ejection fraction (LVEF) $<45 \%$ and decreased exercise tolerance already on digoxin and diuretics with the addition of hydralazine/isosorbide dinitrate to a matched cohort receiving placebo or prazosin to determine the effects on mortality. The V-HEFT study showed that the addition of hydralazine/isosorbide dinitrate had a favorable effect on LV function and mortality, the risk reduction in mortality by 2 years was $34 \%(\mathrm{P}<0.028)$. On the other hand, the mortality in the prazosin group was similar to that in the placebo group (4).

\section{Angiotensin converting enzyme (ACE) inhibitors}

Shortly after V-HEFT trial was published the results of the CONSENSUS trial were published in 1987. Up to that time, the beneficial effects on hemodynamics and symptoms of the ACE inhibitors were widely known but their overall effect on mortality was not established (5). CONSENSUS trial randomized 253 patients to evaluate the effect of enalapril on mortality in patients with NYHA class IV severe heart failure with reduced ejection fraction (HFrEF) in addition to conventional therapy, which included vasodilators and diuretics. Overall, enalapril showed a $40 \%$ reduction in mortality by 6 months and $31 \%$ by 1 year as well as improvements in NYHA class, reduction in the need for other HF medications and reductions in heart size (6). Despite the results of CONSENSUS, the mortality benefit on patients with NYHA II-III mild or moderate HFrEF with LVEF $<35 \%$ was demonstrated by the SOLVD trial. It randomized 2,569 patients who were followed for 4 years (average 41.4 months). The study found that there was a $16 \%$ reduction in the risk of death and an overall $22 \%$ composite risk reduction in deaths and hospitalizations in the patients treated with enalapril (7).

However, up until that time it was not known whether vasodilator therapy with ACE inhibitors vs. hydralazine/ isosorbide dinitrate was superior as both had shown mortality benefits in HFrEF. In the V-HEFT II trial, 804 men with NYHA II-III chronic HF on digoxin and diuretics were randomized to enalapril therapy $v s$. hydralazine/isosorbide dinitrate with a mean follow-up of 2.5 years. Overall, there was a reduction in mortality in patients treated with enalapril of $28 \%$ with $\mathrm{P}=0.016$ that was mainly driven by a reduction in sudden death (8). Hydralazine/isosorbide dinitrate treatment was associated with improvement in body oxygen consumption at peak exercise $(\mathrm{P}<0.05)$. LVEF was noted to increase in both regimens during the 2-year follow-up, but increased more in the first 13 weeks in the hydralazine/isosorbide dinitrate arm.

Current guidelines recommend ACE inhibitors in symptomatic or asymptomatic patients with reduced LVEF (<40\%) (9). Generally started at the lowest-dose and up-titrated every 3 days to the highest tolerated dose. Hypotension and hyperkalemia are the more common limiting factors from achieving the appropriate dose.

\section{Angiotensin II receptor blockers (ARBs)}

Due to the side effect profile of the ACE inhibitors, there is a subgroup of patients whom are unable to receive these medications and thus do not benefit from their proven mortality benefit. For these patients, ARBs may be an alternative. In the ELITE trial, 722 patients aged $\geq 65$ years with NYHA class II-IV HF and LVEF $\leq 40 \%$ were randomized to receive losartan or captopril. Treatment with losartan was associated with a lower incidence of mortality ( $4.8 \%$ vs. $8.7 \%, \mathrm{P}=0.035$ ), no difference in the incidence of renal dysfunction, and a better tolerated side effect profile (10). Given the results of the ELITE trial, ELITE II trial sought to confirm whether losartan was superior to captopril in terms of mortality benefits by randomizing 
3,152 patients aged $\geq 60$ years with NYHA class II-IV HF with LVEF of $\leq 40 \%$ to receive losartan $v$ s. captopril. ELITE II showed that there was no difference in all-cause mortality $(11.7 \%$ vs. $10.4 \%)$ or sudden death/resuscitated arrests $(9.0 \%$ vs. $7.3 \%)$ between the two groups but losartan was better tolerated (11).

Later on, in Val-HeFT trial, 5,010 patients with NYHA class II-IV HF were randomized to receive valsartan $v s$. placebo and the primary outcomes of mortality and combined end point of mortality and morbidity was compared. Treatment with valsartan did not improve overall mortality but had a $13.2 \%$ lower incidence of the combined end point of mortality and morbidity $(\mathrm{P}=0.009)$ that was mainly driven by a decrease in HF hospitalizations (12). A post-hoc analysis also showed that combination of ACE inhibitors, ARBs, and beta-blocker was associated with a greater incidence of adverse effects in the study population. Despite the findings of the Val-HeFT trial, the CHARMAdded trial sought to answer the question of whether dual neurohumoral inhibition of the renin-angiotensinaldosterone system (RAAS) had mortality benefit in chronic HF patients. In this study, 2,548 patients with NYHA class II-IV HF with LVEF $\leq 40 \%$ already treated with ACE inhibitors were randomized to receive candesartan or placebo. Overall treatment with candesartan reduced the incidence of cardiovascular death $(38 \%$ vs. $42 \%, \mathrm{P}=0.011)$ as well as the total number of hospital admissions for CHF (24.2\% vs. $28.0 \%, \mathrm{P}=0.014$ ) (13). However, despite the reduction in cardiovascular mortality and morbidity seen with combined use of ACE inhibitors and ARBs in chronic HF patients, there was a higher incidence of discontinuation of candesartan due to adverse events or abnormal lab values (24\% vs. $18 \%, \mathrm{P}=0.0003)$. As such, dual use of ARBs and ACE inhibitors may have a mortality benefit in chronic HF patients but require close monitoring (9).

\section{Beta-blockers}

One of the first studies to evaluate beta-blockers effect as a class in patients with HF was the MERIT-HF study published in 1999. At the time of the study, shortacting metoprolol tartrate was known to reduce clinical deterioration, improve symptoms, quality of life and EF in patients with dilated cardiomyopathy due to the results of the MDC study (14). Shortly after that extended-release (CR/XL) metoprolol was developed and studied in the MERIT-HF trial where 3,991 patients with NYHA class II-IV symptomatic HF with LVEF $<40 \%$ on optimum standard therapy of ACE inhibitors and diuretics were randomized. There was 34\% reduction in mortality from extended release metoprolol that the study was stopped early. Benefit was mainly driven by a reduction in sudden cardiac death and progressive pump failure regardless of an ischemic $v s$. nonischemic cause for the patient's HF (15).

In CIBIS-II trial, bisoprolol demonstrated reduced allcause mortality by $32 \%$, sudden cardiac death by $45 \%$, and all-cause hospitalizations by $15 \%$. CIBIS-II also attempted to demonstrate non-inferiority of a bisoprolol-first strategy $v s$. an enalapril-first strategy but was unable to do so (16).

Carvedilol had three important trials that led to its approval in HF patients. The U.S. Carvedilol Heart Failure Program was composed of four separate trials that had to be stopped early after an average of 6.5 months because of a significant $65 \%$ reduction in mortality in patients treated with carvedilol (17). COPERNICUS trial randomized 2,289 patients with advanced HF ( $\mathrm{EF}<25 \%)$ to carvedilol $v s$. placebo and found a reduction in mortality of $35 \%$ and a reduction in relative risk of death or HF hospitalization by $31 \%$ (18). Finally, the COMET trial sought to compare the effect of carvedilol $v s$. metoprolol tartrate on allcause mortality, which showed $33 \%$ reduction in all-cause mortality in patients receiving carvedilol (19). As a result of the COMET trial, metoprolol tartrate is not recommended in the treatment of patients with HF.

Under present guidelines, beta blockers should be used in all symptomatic or asymptomatic patients with HFrEF and an EF $<40 \%$ (9). Within the class of beta blockers, metoprolol succinate, carvedilol, and bisoprolol have been extensively studied and shown a mortality benefit.

\section{Mineralocorticoid receptor antagonists (MRAs)}

With more advanced HF, addition of MRAs such as spironolactone or eplerenone may be necessary to improve patient symptoms and survival. The first trial to show benefit of aldosterone antagonists in HF was the RALES trial published in 1999. In this trial, patients with NYHA class III-IV symptoms, EF $<35 \%$ on GDMT of the time which include ACE inhibitors, loop diuretics and digitalis were randomized to spironolactone or placebo. The trial had to be discontinued early after a mean follow-up period of 24 months because of spironolactone's efficacy. There was $30 \%$ reduction in the risk of death, a $35 \%$ reduction in hospitalizations due to worsening HF, and a significant improvement in NYHA class symptoms (20). Of men receiving spironolactone, $10 \%$ reported gynecomastia while 
serious hyperkalemia was minimal in both groups.

Current guideline recommends to use spironolactone in symptomatic patients with NYHA class III-IV and EF $<35 \%$ on GDMT (9). Hyperkalemia is a potential serious adverse effect with use of spironolactone and eplerenone. Potassium levels must be checked on a regular interval once initiated.

\section{Diuretics}

Diuretics help achieve euvolemia in an acute decompensated state and maintain euvolemia in stable chronic $\mathrm{HF}$ patients. To this date there has been no trial that has shown a mortality benefit in patients with HF. Generally, loop diuretics are first line when it comes to treatment compensated and decompensated HF but thiazide and MRAs are also employed. Thiazide diuretics can be effective in early stage HF to maintain euvolemia but generally lose efficacy with worsening renal function or progression of HF. In these scenarios, switching to a loop diuretic tends to be a better option. However, in acute decompensated $\mathrm{HF}$ or chronic advanced $\mathrm{HF}$, thiazide diuretics can be used synergistically with loop diuretics to improve diuresis (9).

The efficacy of bolus intravenous (IV) therapy $v s$. continuous infusion of furosemide in acute decompensated states was evaluated in the DOSE trial. In the trial patients with acute decompensated HF were randomized to receive either IV bolus furosemide twice a day at the dose equivalent to their home dose $v s$. continuous infusion of furosemide at the equivalent home dose or 2.5 times the equivalent home dose. The primary endpoints were the patient's global assessment of symptoms, which was represented as the area under the curve of a visual-analogue scale and change in serum creatinine level in 72 hours. The DOSE trial demonstrated that in patients with decompensated HF there was no significant change in patient's global assessment of symptoms or change in creatinine level when an IV bolus $v s$. continuous infusion of furosemide was used. Patients in the high dose continuous infusion therapy did achieve greater diuresis and improvement in secondary measures such as net fluid loss, change in weight, and area under the curve for dyspnea at 72 hours but also had temporary worsening of their renal function (21).

\section{Digoxin}

Digoxin is a cardiac glycoside that is used as adjunctive therapy in patients whom remain symptomatic despite maximally tolerated medical therapy frequently in the setting of atrial fibrillation. Digoxin was studied in chronic HF patients in the DIG trial in which patients in sinus rhythm with chronic $\mathrm{HF}$ and $\mathrm{EF}<45 \%$ were randomized to receive digoxin $v s$. placebo already treated with diuretics and ACE inhibitors. This trial showed there was no difference in overall mortality but there was a $6 \%$ decrease in hospitalizations for worsening HF. However, in subgroup analysis a dose-dependent increase in mortality was seen with digoxin with the least mortality observed when trough levels in men were between 0.6 to $0.8 \mathrm{ng} / \mathrm{mL}$ (22). The narrow therapeutic window with digoxin and significant drug-drug interactions limit the use of digoxin. The most common side effects that can be seen in digoxin toxicity are cardiac arrhythmias including heart block, neurological complaints including visual changes, altered mental status, confusion and gastrointestinal symptoms that include anorexia, nausea and vomiting. The DIG study was performed prior to the widespread use of ACE inhibitors and beta-blockers, there is no data presently on the effect of adjunctive therapy in patients treated with both ACE inhibitors and beta blockers (23).

\section{Ivabradine}

It has been shown in multiple studies that elevated heart rates are directly related to risk of death, cardiovascular death, or hospital admission for $\mathrm{HF}$ and that reducing baseline heart rate improves outcomes $(24,25)$. In fact, in one study of patients with coronary artery disease and reduced EF, a baseline heart rate above 70 beats per minute was associated with a $34 \%$ increased risk of cardiovascular death and $53 \%$ increase in hospital admissions for HF when compared to similar patients with resting heart rates lower than 70 beats per minute (26). Ivabradine exerts its effects by inhibiting the $\mathrm{I}_{\mathrm{f}}$ current in the sinoatrial (SA) node. As a result, blockade of the f-current in the SA node leads to prolongation of the slow depolarization phase and an overall decrease in resting heart rate (27). The beneficial effects of ivabradine were evaluated in the SHIFT trial that was published in 2010. In this study, symptomatic HFrEF NYHA II-IV patients with an LVEF <35\% in sinus rhythm with a baseline heart rate of $\geq 70 \mathrm{bpm}$ and HF hospitalization within the past year on GDMT (betablockers, ACE inhibitor/ARB, and aldosterone antagonists) were randomly assigned to receive ivabradine to a target dose of $7.5 \mathrm{mg}$ twice daily $v s$. placebo. Ivabradine had an $18 \%$ reduction in the composite primary endpoint of 
cardiovascular death or hospital admission for worsening HF after a mean follow-up period of 22.9 months (28). Furthermore, ivabradine was noted to decrease secondary endpoints of death from HF, hospital admission for HF, and any cardiovascular admission but it did not seem to affect cardiovascular, all-cause deaths, or sudden cardiac death. Ivabradine is recommended in chronic HF patients whom are unable to achieve the target heart rate of $\leq 60 \mathrm{bpm}$ on maximally tolerated beta-blocker therapy or in those patients whom are intolerant to beta-blockers (29).

\section{Angiotensin receptor neprilysin inhibitors (ARNIs)}

Now that the classical GDMT have been discussed, we can turn our focus on the novel therapies from the past few years that have greatly impacted the treatment of chronic HF. There have not been any landmark studies since the advent of the studies that approved beta-blockers and RAAS blockers for use in chronic HF. The ARNIs will be covered briefly here as there is another article in the series that will cover this class of medications in more detail. In brief, ARNIs work by counteracting the chronic neurohormonal activation that is seen in in chronic HF. In particular, neprilysin is responsible for the breakdown of B-type natriuretic peptide (BNP), bradykinin, and adrenomodullin $(22,30,31)$. It is the breakdown of these vasoactive peptides that leads to vasoconstriction, sodium retention and negative remodeling $(32,33)$. However, inhibition of neprilysin leads to elevation in bradykinin levels which leads to serious adverse effects. Initial trials tried to circumvent this by pairing a neprilysin inhibitor with an ACE inhibitor but such a combination was unsuccessful because of the high rate of angioedema observed $(34,35)$. However, combination of neprilysin inhibitor with an ARB had similar rates of angioedema seen with the use of ACE inhibitor alone without compromising the beneficial effects of the neprilysin inhibitor. Sacubitril-valsartan has been evaluated in two landmark trials: PARADIGM-HF and PIONEER$\mathrm{HF}$, which have led to its approval in the treatment of chronic HF.

PARADIGM-HF that was published in 2014 involved 8,399 patients with NYHA II-IV chronic HF and reduced LVEF $\leq 40 \%$. Treatment with sacubitril-valsartan lead to $21.8 \%$ reduction in composite endpoint of risk of death from cardiovascular causes or hospitalization compared to $26.5 \%$ with enalapril group $(\mathrm{P}<0.001)(36)$. Sacubitril-valsartan was also superior to enalapril at reducing the symptoms of
HF. However, PARADIGM-HF only evaluated patients with stable chronic HF and efficacy of the medication in acute decompensated HF was not known. PIONEERHF that was published in 2019 sought to evaluate if there was a benefit of starting ARNI after stabilization from an acute decompensated HF hospitalization. The ARNI was initiated in patients hospitalized for HF with LVEF $\leq 40 \%$ and an N-terminal proBNP (NT-proBNP) concentration $\geq 1,600 \mathrm{pg} / \mathrm{mL}$ or a BNP concentration $\geq 400 \mathrm{pg} / \mathrm{mL}$ in comparison to enalapril. Sacubitril-valsartan was superior to enalapril at reducing NT-proBNP levels as well as reducing the rate of $\mathrm{HF}$ rehospitalization at 8 weeks when compared to enalapril alone (37).

ARNIs are presently a class I indication to start patients on with $\mathrm{HF}$ with an $\mathrm{EF}<40 \%$ as well as chronic $\mathrm{HF}$ patients whom remain symptomatic with NYHA class II-III symptoms. ARNIs are generally well-tolerated but can be associated with renal dysfunction, hyperkalemia, and symptomatic hypotension but with the exception of symptomatic hypotension, the rates of these adverse reactions are not greater than those observed when ACE inhibitors are taken by themselves. Symptomatic hypotension is the adverse event most commonly seen with the use of ARNI but given the significant benefit of this medication, it is recommended that the dose of the diuretic being taken by the patient be reduced instead of stopping the ARNI altogether (29).

\section{Vericiguat}

Besides inhibition of neprilysin and the RAAS there are now new medications currently under investigation that have potential benefits in patients with chronic HFrEF. One of these drugs is vericiguat which is a novel oral guanylate cyclase stimulator. Chronic HF is associated with endothelial dysfunction and the production of reactive oxygen species that inherently reduce the availability of nitric oxide and thus the availability of soluble guanylate cyclase and cyclic GMP generation (cGMP) (38). Increased cGMP inhibits the entry of calcium in vascular smooth muscle cells, activates $\mathrm{K}^{+}$channels leading to hyperpolarization and relaxation and stimulates a cGMPdependent protein kinase that then activates myosin light chain phosphatase. As a result, increased cGMP leads to many beneficial effects such as vasodilation, anti-thrombotic, anti-inflammatory and anti-proliferative effects (39). Vericiguat directly stimulates soluble guanylate cyclase via a binding site independent of nitric oxide and also sensitizes 
guanylate cyclase to endogenously produced nitric oxide. It is via these mechanisms that vericiguat is thought to exert its beneficial effects in HF patients (39). The efficacy and safety of vericiguat in chronic HF patients with reduced EF were evaluated in the VICTORIA trial that was recently published in May 2020. The trial enrolled 5,050 patients who were at least 18 years of age with chronic HF on GDMT with NYHA class II-IV symptoms and $\mathrm{EF}<45 \%$. Patients were randomized to receive vericiguat (target dose $10 \mathrm{mg}$ daily) or placebo. The primary outcome was composite of death from cardiovascular causes or first hospitalization. Vericiguat had a $10 \%$ relative difference in the primary composite endpoint of death from cardiovascular cause or hospitalization for HF after a median follow-up period of 10.8 months (40). Though there was a higher rate of syncope and symptomatic hypotension the overall frequency of adverse events was similar $(\mathrm{P}=0.30$ and $\mathrm{P}=0.12$, respectively). There were also reports of slightly lower but clinically insignificant hemoglobin levels at the 16-week follow-up period. In summary, vericiguat has potential and shown benefit in reducing death from cardiovascular causes or first HF hospitalization in patients with chronic HF on GDMT who have signs of worsening HF. More studies are needed to further evaluate long-term efficacy of the medication as well as to evaluate overall improvement in heart function and prevention of worsening pump failure.

\section{SGLT2 inhibitors}

Another medication group that is also showing promise in the treatment of patients with chronic HF is the SGLT2 inhibitors. It is well known that type 2 diabetes mellitus (T2DM) is a potential risk factor for the development of $\mathrm{HF}$ and in those with an established diagnosis of HF, T2DM is a common comorbidity which makes treatment of the two simultaneous conditions vital yet challenging. The SGLTs are proteins found within the proximal convoluted tubules of the kidneys and play a vital role in the glucose homeostasis. SGLT2 is the major protein involved in $90 \%$ of the reabsorption of glucose from the glomerular filtrate. SGLT2 inhibitors function by competitively and selectively inhibiting the SGLT2 protein, which in turn leads to decreased reabsorption of glucose into the blood and glucosuria. Furthermore, unlike many of the other diabetic medications, SGLT2 inhibitors function independently of insulin secretion and sensitivity which allows these medications to exert a greater glucosuric effect with higher blood glucose levels. Overall, the SGLT2 inhibitors are well tolerated and the more common adverse reactions are mycotic infections and urinary tract infections. Less common but serious adverse reactions include the risk for diabetic ketoacidosis as well as an increased risk of lower limb amputation, mainly of the toes, and a possible risk for Fournier gangrene (41).

The potential benefit of SGLT2 inhibitors on cardiovascular mortality has been demonstrated in the CANVAS and EMPA-REG OUTCOME trials. CANVAS demonstrated that patients with T2DM with established cardiovascular disease or those at high risk for cardiovascular disease treated with canagliflozin had a lower risk of death from cardiovascular causes, nonfatal myocardial infarction (MI) or nonfatal stroke compared to placebo $(26.9$ vs. 31.5 events per 1,000 patient-years, hazard ratio $0.86, \mathrm{P}<0.001$ ) but a greater risk of lower limb amputation (6.3 vs. 3.4 events per 1,000 patient-years, hazard ratio 1.97) (42). EMPA-REG OUTCOME study further enforced the benefit of SGLT2 inhibitors (empagliflozin) on cardiovascular outcomes since it showed that patients with T2DM at high risk for cardiovascular events had a lower rate of the primary composite outcome of death from cardiovascular causes, nonfatal MI, or nonfatal stroke as well as risk of death from any cause and hospitalization for $\mathrm{HF}$ in patients treated with empagliflozin when compared to patients treated with placebo (43). However, despite the lower risk for HF hospitalization seen in both CANVAS and EMPA-REG OUTCOME, there was no real data available on the potential benefits of SGLT2 inhibitors on morbidity and mortality in patients with established HF.

The DECLARE-TIMI 58 trial evaluated the effect of dapagliflozin on the composite end point of cardiovascular death and hospitalization for HF in a diverse patient population with T2DM. Treatment with dapagliflozin reduced the risk of cardiovascular death or hospitalization for $\mathrm{HF}$ to a greater extent in patients with reduced $\mathrm{EF}$ (EF $<45 \%$ ) than in those patients that had no history of reduced EF. As such, besides deriving a mortality benefit and decreased HF hospitalization in patients with known cardiovascular disease or at high risk for cardiovascular disease, the SGLT2 inhibitors also seem to be beneficial in patients with chronic HF with reduced EF on GDMT (44). Although, the DECLARE-TIMI 58 was not a dedicated HF study, the results have offered valuable insight into the potential benefit of this class of medication in patients with chronic HF and concurrent T2DM.

That being said, the effect of SGLT2 inhibition on HF 
hospitalizations and mortality in patients with chronic $\mathrm{HF}$ regardless of the presence or absence of T2DM was not known until the results of the DAPA-HF trial were published in late 2019. In this study, patients with at least 18 years of age and an EF $\leq 40 \%$ with NYHA class IIIV symptoms were studied to determine if treatment with dapagliflozin had any impact on the primary endpoint of worsening HF or death from cardiovascular causes. After a follow-up period of 18.2 months, the primary composite endpoint of worsening HF, defined as hospitalization or an urgent visit resulting in IV therapy for HF, or death from cardiovascular causes occurred in $16.3 \%$ in the dapagliflozin group and $21.2 \%$ in the placebo group (45). The incidence of the secondary composite outcome of hospitalization for HF or death from cardiovascular causes was also found to be lower in the dapagliflozin group than in the placebo arm (44). Interestingly, the effect of dapagliflozin on the combined primary outcome was consistent regardless of the presence or absence of T2DM but it was noted that patients with NYHA class III-IV derived less of a benefit when compared to those patients with NYHA class II symptoms. Furthermore, because the benefit of SGLT2 inhibitors was seen shortly after the randomization period and in the $55 \%$ of the patients in the study without concurrent T2DM it is believed that the effect of dapagliflozin on the composite endpoint may be due to other mechanisms than just the glucose lowering mechanism. Overall, the DAPA-HF study provided valuable insight as to the potential beneficial effects of SGLT2 inhibitors on patients with chronic HF regardless of the presence of T2DM. Although Cardiologists may be apprehensive of starting a hypoglycemic agent in patients without evidence of hyperglycemia, the DAPA-HF study showed that in patients treated with dapagliflozin the incidence of hypoglycemia or diabetic ketoacidosis was not very frequent and only observed in patients with T2DM.

Although the mechanism for the cardioprotective effect of the SGLT2 is incompletely understood, this class of medication promotes natriuresis and osmotic diuresis causing contraction in plasma volume and preload, decrease blood pressure, arterial stiffness, and afterload which is thought to improve subendocardial blood flow in those patients with HF (46). Therefore, given the recent influx of studies evaluating the effect of SGLT2 inhibitors on chronic HF patients, it can be said that overall there is a mortality as well as protective effect from worsening pump failure in these patients regardless of the diagnosis of diabetes without a significantly increased risk of adverse effects that would make these medications restrictive in the non-diabetic patient with HF.

Please see Table 1 for a list of the drug classes currently in use for patients with HFrEF $(9,28,40,45)$.

\section{Iron replacement in HFrEF}

Of the many therapies currently being evaluated for the treatment of chronic HF patients, iron replacement needs to be mentioned. Iron deficiency can be commonly seen in patients with chronic HF and has been associated with poor prognosis as well as decreased exercise capacity and worse quality of life $(47,48)$.

Three trials have evaluated the effect of iron repletion in ferropenic patients with $\mathrm{HF}$ and their effects on various variables such as exercise capacity, ventilator efficiency and 6-minute walk-distance. EFFECT-HF study evaluated the effect of ferric carboxymaltose $v s$. usual care on exercise capacity. After 24 weeks of treatment, ferric carboxymaltose was associated with a higher peak oxygen consumption than the usual care arm (49). FERRIC-HF II was another trial that sought to evaluate the effects of iron repletion with iron isomaltoside in patients with symptomatic HF with reduced LVEF on phosphocreatine recovery halftime on dynamic ${ }^{31} \mathrm{P}$ magnetic resonance spectroscopy at submaximal exercise. Repletion with iron isomaltoside was associated with a shorter phosphocreatine time signifying improved mitochondrial oxidative function (50). Lastly, IRONOUT HF evaluated the effect of oral iron repletion with iron polysaccharide on oxygen uptake in patients with chronic HF and reduced LVEF. After a follow-up period of 16 weeks, iron repletion with iron polysaccharide had no effect on peak oxygen consumption, ventilator efficiency, 6-minute walk distance or HF symptoms when compared to placebo (51). These studies have offered some valuable insight about potential benefits of iron repletion in chronic HF patients with reduced LVEF whom are ferropenic but more studies and data are needed to assess the true effect of iron repletion on mortality and symptoms in HF patients. Presently, it is a class IIb indication for IV iron replacement to improve functional status and quality of life in chronic HF patients (29).

\section{Precision medicine}

A rapidly expanding area of research in the realm of $\mathrm{HF}$ is precision medicine in which the "omics"genomics, pharmacogenomics, epigenomics, proteomics, metabolomics, and microbiomics of $\mathrm{HF}$ and their impact 
Table 1 Current GDMT for patients with HFrEF $(9,29,40,45)$

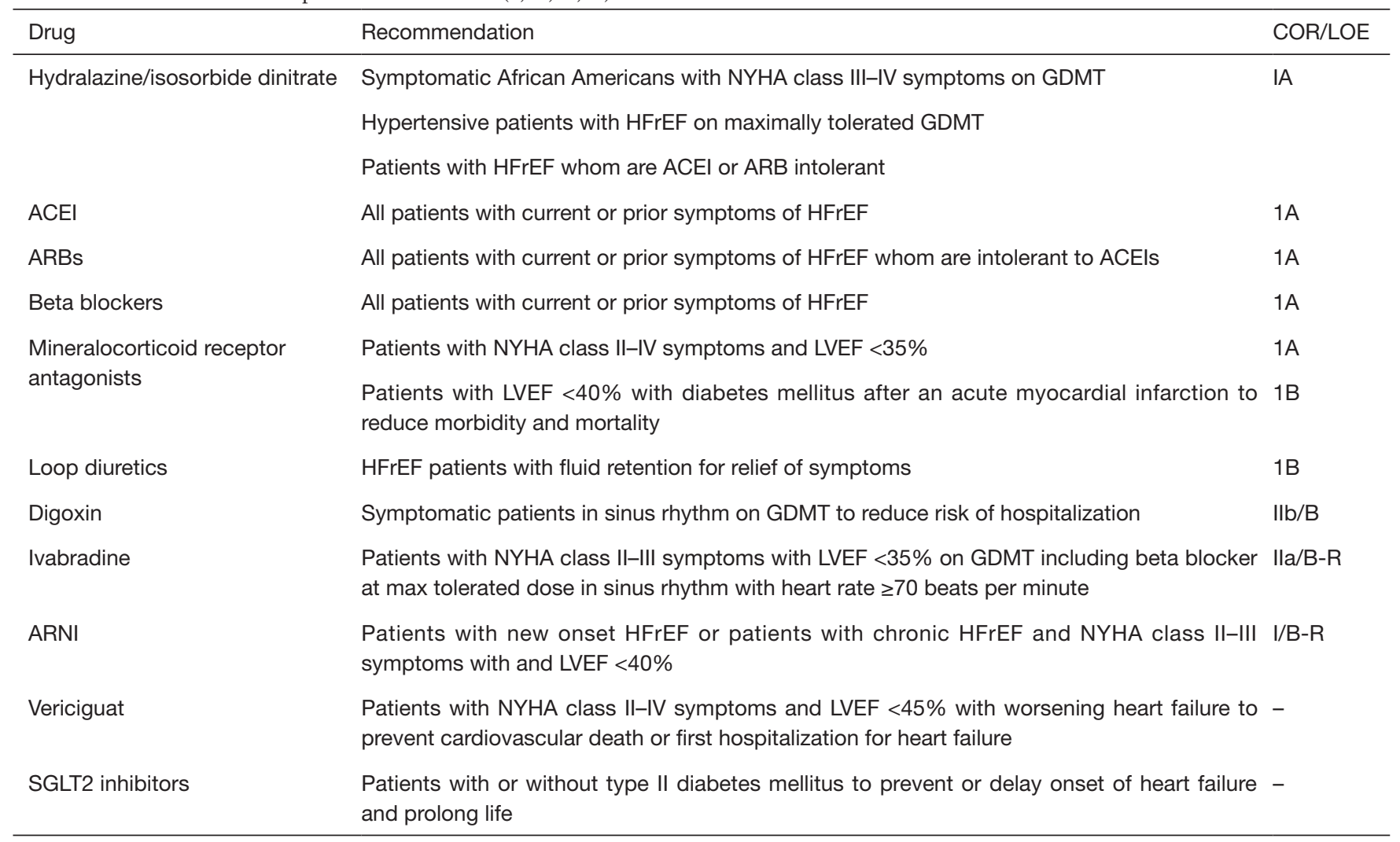

COR, class of recommendation; LOE, level of evidence; GDMT, guideline directed medical therapy; HFrEF, heart failure with reduced ejection fraction; ACEI, angiotensin converting enzyme inhibitor; ARB, angiotensin II receptor blocker; LVEF, left ventricular ejection fraction; ARNI, angiotensin receptor neprilysin inhibitor.

on the development, progression, and treatment are being evaluated. For instance, there is mounting evidence that development of HF may have some degree of genetic inheritance from the cumulative effect of multiple variants in multiple genes each exerting an effect on the potential development of the clinical syndrome (52). Furthermore, substantial work has also been performed in pharmacogenomics or the role of inheritance in observed drug responses. The more widely studied drug class are beta-blockers where genetic variations in the CYP2D6 enzyme can lead to variance in the metabolism of the drugs and therefore differences in clinical response to the medication (52). Although promising, application to clinical practice has been limited by numerous variables including but not limited to lack of replication and small study sample sizes. Another promising area of research is epigenomics or the study of changes in gene expression unrelated to changes in DNA sequence (52). DNA methylation, histone modification and non-coding
RNAs have been implicated in the pathophysiology of $\mathrm{HF}$ and are potential targets for novel HF therapies. A large effort is also being placed in the area of proteomics particularly in the search for new biomarkers that can help detect the presence of disease, severity, risk of future events, and guide therapy or using already discovered markers such as NT-proBNP to guide therapy (52). Metabolomics is another area of research in HF since it has been shown that myocardium is capable of utilizing many different energy substrates to meet its metabolic demand. Research is currently underway with the use of magnetic resonance imaging (MRI) and mass spectrometry to quantify variations in myocardial metabolism to predict, detect, and evaluate prognosis of HF (52). Lastly, with microbiomics, it has been postulated that alterations in gut microbiota may be the result of decreased intestinal mucosal $\mathrm{pH}$ as a result from low cardiac output in the setting of HF. This leads to increased growth of pathogenic bacteria and may be related to the increased 
levels of inflammatory markers observed in patients with advanced HF. Increased growth of pathogenic bacteria also leads to elevated circulating metabolites that can potentially be used as biomarkers for patients with $\mathrm{HF}$ and currently an area of intense research. Although "omics" in HF presently has little clinical applications, the vast work being done in these areas will likely lead to the development of novel therapies for $\mathrm{HF}$ and potentially for the individualization of HF therapy in the foreseeable future.

\section{Conclusions}

HF is impactful in the field of Cardiology and recent advances in the treatment of $\mathrm{HF}$ have led to improved morbidity and mortality in patients with reduced LVEF. HF continues to be an ever-evolving field and hopefully as more studies and data are collected, there can be a trend towards improved patient outcomes and overall decreased clinical burden for both patients and clinicians.

\section{Acknowledgments}

Funding: None.

\section{Footnote}

Provenance and Peer Review: This article was commissioned by the Guest Editors (Dr. Debabrata Mukherjee and Dr. Jose B. Cruz Rodriguez) for the series "Heart Failure Update and Advances in 2021" published in Annals of Translational Medicine. The article has undergone external peer review.

Peer Review File: Available at http://dx.doi.org/10.21037/ atm-20-4640

Conflicts of Interest: All authors have completed the ICMJE uniform disclosure form (available at http://dx.doi. org/10.21037/atm-20-4640). The series "Heart Failure Update and Advances in 2021" was commissioned by the editorial office without any funding or sponsorship. The authors have no other conflicts of interest to declare.

Ethical Statement: The authors are accountable for all aspects of the work in ensuring that questions related to the accuracy or integrity of any part of the work are appropriately investigated and resolved.
Open Access Statement: This is an Open Access article distributed in accordance with the Creative Commons Attribution-NonCommercial-NoDerivs 4.0 International License (CC BY-NC-ND 4.0), which permits the noncommercial replication and distribution of the article with the strict proviso that no changes or edits are made and the original work is properly cited (including links to both the formal publication through the relevant DOI and the license). See: https://creativecommons.org/licenses/by-nc-nd/4.0/.

\section{References}

1. Ponikowski P, Voors AA, Anker SD, et al. 2016 ESC Guidelines for the diagnosis and treatment of acute and chronic heart failure: The Task Force for the diagnosis and treatment of acute and chronic heart failure of the European Society of Cardiology (ESC)Developed with the special contribution of the Heart Failure Association (HFA) of the ESC. Eur Heart J 2016;37:2129-200.

2. Virani SS, Alonso A, Benjamin EJ, et al. Heart Disease and Stroke Statistics-2020 Update: A Report From the American Heart Association. Circulation 2020;141:e139-e596.

3. Braunwald E, Bonow R. Braunwald's Heart Disease. 9th edition. Philadelphia: Saunders, 2012:487-94.

4. Cohn JN, Archibald DG, Ziesche S, et al. Effect of vasodilator therapy on mortality in chronic congestive heart failure. N Engl J Med 1986;314:1547-52.

5. Captopril Multicenter Research Group. A placebocontrolled trial of captopril in refractory chronic congestive heart failure. J Am Coll Cardiol 1983;2:755-63.

6. CONSENSUS Trial Study Group. Effects of enalapril on mortality in severe congestive heart failure. Results of the Cooperative North Scandinavian Enalapril Survival Study (CONSENSUS). N Engl J Med 1987;316:1429-35.

7. SOLVD Investigators, Yusuf S, Pitt B, et al. Effect of enalapril on survival in patients with reduced left ventricular ejection fractions and congestive heart failure. N Engl J Med 1991;325:293-302.

8. Cohn JN, Johnson G, Ziesche S, et al. A comparison of enalapril with hydralazine-isosorbide dinitrate in the treatment of chronic congestive heart failure. $\mathrm{N} \mathrm{Engl} \mathrm{J}$ Med 1991;325:303-10.

9. Yancy CW, Jessup M, Bozkurt B, et al. 2013 ACCF/AHA guideline for the management of heart failure: a report of the American College of Cardiology Foundation/American Heart Association Task Force on Practice Guidelines. J 
Am Coll Cardiol 2013;62:e147-239.

10. Pitt B, Segal R, Martinez FA, et al. Randomised trial of losartan versus captopril in patients over 65 with heart failure (Evaluation of Losartan in the Elderly Study, ELITE). Lancet 1997;349:747-52.

11. Pitt B, Poole-Wilson PA, Segal R, et al. Effect of losartan compared with captopril on mortality in patients with symptomatic heart failure: randomised trial-the Losartan Heart Failure Survival Study ELITE II. Lancet 2000;355:1582-7.

12. Cohn JN, Tognoni G. A randomized trial of the angiotensin-receptor blocker valsartan in chronic heart failure. N Engl J Med 2001;345:1667-75.

13. McMurray JJ, Ostergren J, Swedberg K, et al. Effects of candesartan in patients with chronic heart failure and reduced left-ventricular systolic function taking angiotensin-converting-enzyme inhibitors: the CHARMAdded trial. Lancet 2003;362:767-71.

14. Waagstein F, Hjalmarson A, Swedberg K, et al. Beneficial effects of metoprolol in idiopathic dilated cardiomyopathy. Lancet 1993;342:1441-6.

15. Effect of metoprolol CR/XL in chronic heart failure: Metoprolol CR/XL Randomised Intervention Trial in Congestive Heart Failure (MERIT-HF). Lancet 1999;353:2001-7.

16. The Cardiac Insufficiency Bisoprolol Study II (CIBIS-II): a randomised trial. Lancet 1999;353:9-13.

17. Packer M, Bristow MR, Cohn JN, et al. The effect of carvedilol on morbidity and mortality in patients with chronic heart failure. N Engl J Med 1996;334:1349-55.

18. Packer M, Fowler MB, Roecker EB, et al. Effect of carvedilol on the morbidity of patients with severe chronic heart failure: results of the carvedilol prospective randomized cumulative survival (COPERNICUS) study. Circulation 2002;106:2194-9.

19. Poole-Wilson PA, Swedberg K, Cleland JG, et al. Comparison of carvedilol and metoprolol on clinical outcomes in patients with chronic heart failure in the Carvedilol Or Metoprolol European Trial (COMET): randomised controlled trial. Lancet 2003;362:7-13.

20. Pitt B, Zannad F, Remme WJ, et al. The effect of spironolactone on morbidity and mortality in patients with severe heart failure. N Engl J Med 1999;341:709-17.

21. Felker GM, Lee KL, Bull DA, et al. Diuretic strategies in patients with acute decompensated heart failure. $\mathrm{N}$ Engl J Med 2011;364:797-805.

22. Cruden NL, Fox KA, Ludlam CA, et al. Neutral endopeptidase inhibition augments vascular actions of bradykinin in patients treated with angiotensin-converting enzyme inhibition. Hypertension 2004;44:913-8.

23. Digitalis Investigation Group. The effect of digoxin on mortality and morbidity in patients with heart failure. $\mathrm{N}$ Engl J Med 1997;336:525-33.

24. Pocock SJ, Wang D, Pfeffer MA, et al. Predictors of mortality and morbidity in patients with chronic heart failure. Eur Heart J 2006;27:65-75.

25. Flannery G, Gehrig-Mills R, Billah B, et al. Analysis of randomized controlled trials on the effect of magnitude of heart rate reduction on clinical outcomes in patients with systolic chronic heart failure receiving beta-blockers. Am J Cardiol 2008;101:865-9.

26. Fox K, Ford I, Steg PG, et al. Heart rate as a prognostic risk factor in patients with coronary artery disease and left-ventricular systolic dysfunction (BEAUTIFUL): a subgroup analysis of a randomised controlled trial. Lancet 2008;372:817-21.

27. Dobre D, Borer JS, Fox K, et al. Heart rate: a prognostic factor and therapeutic target in chronic heart failure. The distinct roles of drugs with heart rate-lowering properties. Eur J Heart Fail 2014;16:76-85.

28. Swedberg K, Komajda M, Böhm M, et al. Ivabradine and outcomes in chronic heart failure (SHIFT): a randomised placebo-controlled study. Lancet 2010;376:875-85. Erratum in: Lancet 2010;376:1988.

29. Yancy CW, Jessup M, Bozkurt B, et al. 2017 ACC/ AHA/HFSA focused update of the 2013 ACCF/AHA guideline for the management of heart failure: a report of the American College of Cardiology/American Heart Association Task Force on Clinical Practice Guidelines and the Heart Failure Society of America. J Am Coll Cardiol 2017;70:776-803.

30. Rademaker MT, Charles CJ, Espiner EA, et al. Neutral endopeptidase inhibition: augmented atrial and brain natriuretic peptide, haemodynamic and natriuretic responses in ovine heart failure. Clin Sci (Lond) 1996;91:283-91.

31. Wilkinson IB, McEniery CM, Bongaerts KH, et al. Adrenomedullin (ADM) in the human forearm vascular bed: effect of neutral endopeptidase inhibition and comparison with proadrenomedullin NH2-terminal 20 peptide (PAMP). Br J Clin Pharmacol 2001;52:159-64.

32. Maric $\mathrm{C}$, Zheng $\mathrm{W}$, Walther T. Interactions between angiotensin 11 and atrial natriuretic peptide in renomedullary interstitial cells: the role of neutral endopeptidase. Nephron Physiol 2006;103:p149-56.

33. Kuhn M. Molecular physiology of natriuretic peptide 
signalling. Basic Res Cardiol 2004;99:76-82.

34. Kostis JB, Packer M, Black HR, et al. Omapatrilat and enalapril in patients with hypertension: the Omapatrilat Cardiovascular Treatment vs. Enalapril (OCTAVE) trial. Am J Hypertens 2004;17:103-11.

35. Packer M, Califf RM, Konstam MA, et al. Comparison of omapatrilat and enalapril in patients with chronic heart failure: the Omapatrilat Versus Enalapril Randomized Trial of Utility in Reducing Events (OVERTURE). Circulation 2002;106:920-6.

36. McMurray JJ, Packer M, Desai AS, et al. Angiotensinneprilysin inhibition versus enalapril in heart failure. $\mathrm{N}$ Engl J Med 2014;371:993-1004.

37. Velazquez EJ, Morrow DA, DeVore AD, et al. Angiotensin-neprilysin inhibition in acute decompensated heart failure. N Engl J Med 2019;380:539-48.

38. Follmann M, Ackerstaff J, Redlich G, et al. Discovery of the soluble guanylate cyclase stimulator vericiguat (BAY 1021189) for the treatment of chronic heart failure. J Med Chem 2017;60:5146-61.

39. Stasch JP, Pacher P, Evgenov OV. Soluble guanylate cyclase as an emerging therapeutic target in cardiopulmonary disease. Circulation 2011;123:2263-73.

40. Armstrong PW, Pieske B, Anstrom KJ, et al. Vericiguat in patients with heart failure and reduced ejection fraction. $\mathrm{N}$ Engl J Med 2020;382:1883-93.

41. Tentolouris A, Vlachakis P, Tzeravini E, et al. SGLT2 Inhibitors: A Review of Their Antidiabetic and Cardioprotective Effects. Int J Environ Res Public Health 2019;16:2965.

42. Neal B, Perkovic V, Mahaffey KW, et al. Canagliflozin and cardiovascular and renal events in type 2 diabetes. N Engl J Med 2017;377:644-57.

43. Zinman B, Wanner C, Lachin JM, et al. Empagliflozin, cardiovascular outcomes, and mortality in type 2 diabetes. N Engl J Med 2015;373:2117-28.

Cite this article as: Espinoza C, Alkhateeb H, Siddiqui T. Updates in pharmacotherapy of heart failure with reduced ejection fraction. Ann Transl Med 2021;9(6):516. doi: 10.21037/ atm-20-4640
44. Kato ET, Silverman MG, Mosenzon O, et al. Effect of dapagliflozin on heart failure and mortality in type 2 diabetes mellitus. Circulation 2019;139:2528-36.

45. McMurray JJ, Solomon SD, Inzucchi SE, et al. Dapagliflozin in patients with heart failure and reduced ejection fraction. N Engl J Med 2019;381:1995-2008.

46. Lytvyn Y, Bjornstad P, Udell JA, et al. Sodium glucose cotransporter-2 inhibition in heart failure: potential mechanisms, clinical applications, and summary of clinical trials. Circulation 2017;136:1643-58.

47. Jankowska EA, Rozentryt P, Witkowska A, et al. Iron deficiency: an ominous sign in patients with systolic chronic heart failure. Eur Heart J 2010;31:1872-80.

48. Enjuanes C, Klip IT, Bruguera J, et al. Iron deficiency and health-related quality of life in chronic heart failure: results from a multicenter European study. Int J Cardiol 2014;174:268-75.

49. van Veldhuisen DJ, Ponikowski P, van der Meer P, et al. Effect of ferric carboxymaltose on exercise capacity in patients with chronic heart failure and iron deficiency. Circulation 2017;136:1374-83.

50. Charles-Edwards G, Amaral N, Sleigh A, et al. Effect of iron isomaltoside on skeletal muscle energetics in patients with chronic heart failure and iron deficiency: FERRICHF II Randomized Mechanistic Trial. Circulation 2019;139:2386-98.

51. Lewis GD, Malhotra R, Hernandez AF, et al. Effect of oral iron repletion on exercise capacity in patients with heart failure with reduced ejection fraction and iron deficiency: the IRONOUT HF randomized clinical trial. JAMA 2017;317:1958-66.

52. Cresci S, Pereira NL, Ahmad F, et al. Heart Failure in the Era of Precision Medicine: A Scientific Statement From the American Heart Association. Circ Genom Precis Med 2019;12:458-85. 